\title{
Technè
}

La science au service de l'histoire de l'art et de la préservation des biens culturels

$44 \mid 2016$

Archives de l'humanité : les restes humains patrimonialisés

\section{Datation par le carbone 14 et restes humains}

Une étude de cas : la momie dorée de Dunkerque

Carbon-14 dating of human remains. A case-study: the gilded mummy in

Dunkerque museum

\section{Pascale Richardin et Magali Coudert}

\section{OpenEdition}

Journals

Édition électronique

URL : http://journals.openedition.org/techne/1099

DOI : 10.4000/techne.1099

ISSN : 2534-5168

Éditeur

C2RMF

Édition imprimée

Date de publication : 1 novembre 2016

Pagination : 74-78

ISBN : 978-2-7118-6339-6

ISSN : $1254-7867$

Référence électronique

Pascale Richardin et Magali Coudert, «Datation par le carbone 14 et restes humains », Technè [En ligne], 44 | 2016, mis en ligne le 19 décembre 2019, consulté le 23 juillet 2020. URL : http:// journals.openedition.org/techne/1099; DOI : https://doi.org/10.4000/techne.1099

La revue Technè. La science au service de l'histoire de l'art et de la préservation des biens culturels est mise à disposition selon les termes de la Licence Creative Commons Attribution - Pas d'Utilisation Commerciale - Pas de Modification 4.0 International. 


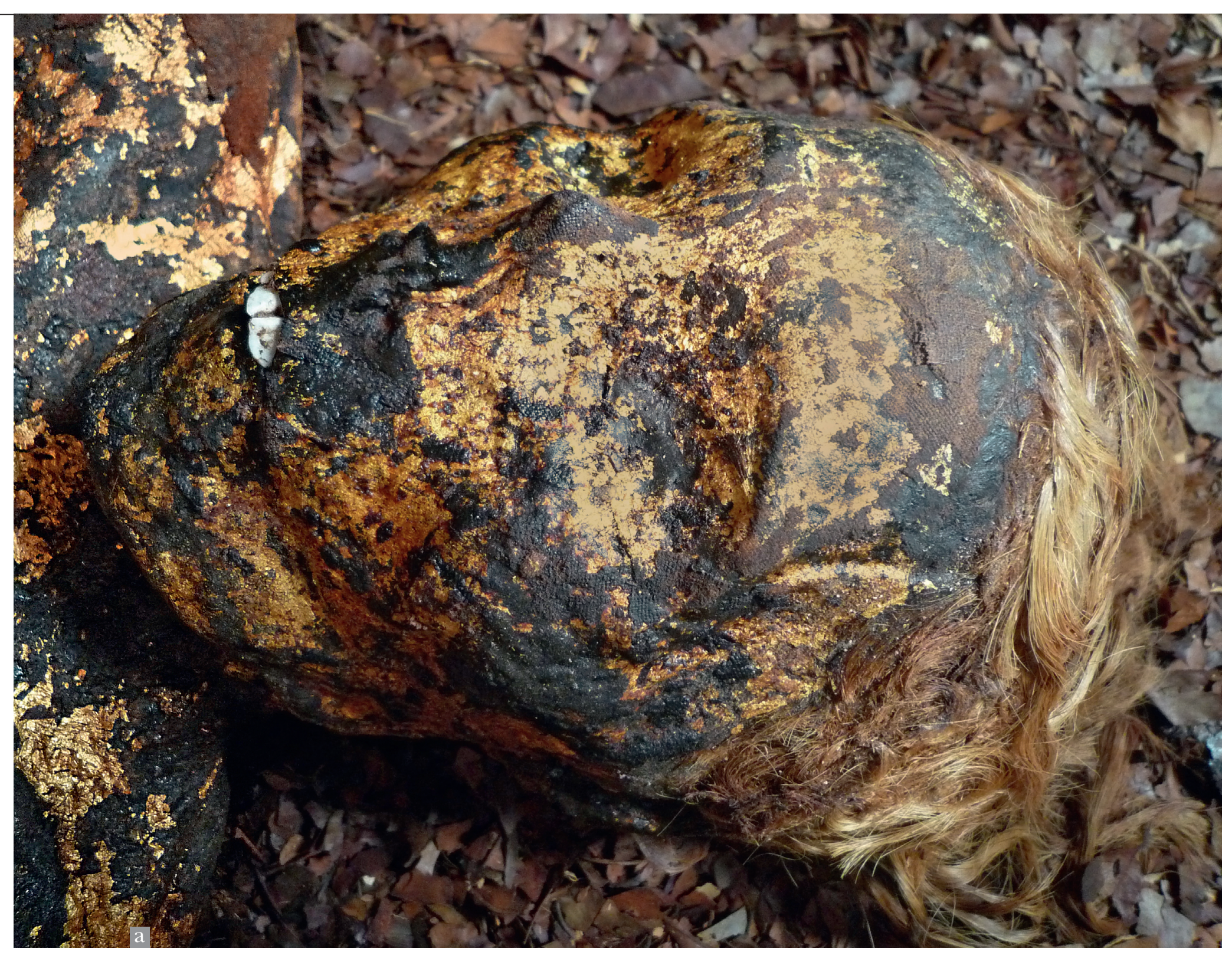

Fig. 1 a. La momie dorée de Dunkerque, la tête laisse apparaître ses cheveux blonds et les feuilles d'or appliquées sur le visage. (c) C2RMF/P. Richardin. 
Pascale Richardin

Magali Coudert

\section{Datation par le carbone 14 et restes humains. \\ Une étude de cas : la momie dorée de Dunkerque}

Carbon-14 dating of human remains.

A case-study: the gilded mummy

in Dunkerque museum

Résumé. Cet article présente la datation par le carbone 14, appliquée à différents échantillons (textiles, végétaux, cheveux) prélevés sur la momie égyptienne dorée conservée au musée de Dunkerque. Les résultats illustrent bien et permettent de valider les protocoles de préparation des échantillons pour la datation: de l'étape d'extraction des matières organiques exogènes à celle de la kératine des cheveux. En effet, ces protocoles doivent être rigoureusement contrôlés, car ils sont essentiels à l'obtention de résultats fiables et reproductibles.

Mots-clés. Datation par le carbone 14, préparation des échantillons, cheveux de momies, Égypte, Dunkerque.
Abstract. This article presents the radiocarbon dating applied on different samples (textiles, plants, hair) taken on the golden Egyptian mummy, preserved in the museum of Dunkerque, France. The results illustrate and validate the sample preparation protocols used: from the extraction steps of exogenous organic matter to those of the hair keratin. Indeed, these protocols must be strictly controlled, because they are essential to obtain reliable and reproducible results.

Keywords. Radiocarbon dating, samples preparation, mummy hair, Egypt, Dunkerque.

\section{Introduction}

La datation directe des restes humains est essentielle pour replacer les individus dans un cadre chronologique et culturel fiable, surtout s'ils sont hors contexte historique ou archéologique, comme beaucoup de momies de collections muséales. La datation par le carbone 14, qui permet de répondre à bien des questionnements à ce sujet, est généralement réalisée sur le collagène des os ${ }^{1}$ ou sur d'autres tissus biologiques, comme l'émail dentaire ${ }^{2}$. Mais cette démarche reste limitée, voire inconcevable, surtout quand il s'agit d'étudier des momies conservées dans des musées ${ }^{3}$. En effet, l'échantillonnage nécessaire serait beaucoup trop invasif.

C'est pourquoi un protocole particulier pour la préparation des échantillons de cheveux, basé sur l'extraction sélective de la kératine du cortex, a été mis en place ${ }^{4}$ et ne nécessite qu'une vingtaine de milligrammes d'échantillon brut $^{5}$. Pour les os, par comparaison, une quantité allant de 200 à 500 mg est nécessaire afin d'obtenir une date pertinente. De plus, si aucun tissu biologique n'est accessible, des échantillons prélevés sur les momies, comme les textiles de linceul ou des bandelettes de momies égyptiennes ${ }^{6}$, peuvent être datés, même s'ils ne permettent pas réellement de dater le corps. Cependant, ils sont souvent imprégnés de grandes quantités de matière organique, baumes de momification ou fluides corporels issus de la décomposition du corps, et leur présence peut fortement modifier les résultats de datation. C'est pourquoi, la préparation des échantillons nécessite des étapes indispensables d'extraction de ces matières exogènes. Ces protocoles doivent être rigoureusement contrôlés, car ils sont essentiels à l'obtention de résultats fiables et reproductibles. Nous allons montrer, sur la momie dorée de Dunkerque, comment ils ont été appliqués avec succès sur les cheveux et le matériel funéraire associé.

À la suite de l'Expédition d'Égypte de Bonaparte à la fin du XVIII ${ }^{\mathrm{e}}$ siècle et après la décision ministérielle de mettre en dépôt les objets collectés en Égypte par Albert Gayet (1856-1916) sur le site d'Antinoé, de nombreux musées français se sont dotés de collections égyptologiques. La ville de Dunkerque fera quelques acquisitions dès 1838, mais il faudra attendre 1907 pour que la collection prenne une réelle importance avec l'arrivée d'une «momie dorée ", désignée par le nom de "Ounnout » (l'«astrologue »). Cette momie aux cheveux blonds (fig. $1 \mathrm{a}, \mathrm{b}$ ), allongée sur un lit de feuilles, dont le corps est recouvert de nombreuses feuilles d'or ${ }^{7}$, a fait partie du corpus d'étude lors du projet de publication ${ }^{8}$, porté par le Service du Récolement des Dépôts antiques et des Arts de l'Islam (SRDAI) du musée du Louvre sur certaines œuvres coptes issues des fouilles du site d'Antinoé. Parmi les analyses scientifiques réalisées, la datation était l'un des volets importants du projet ${ }^{9}$.

Pascale Richardin, ingénieure de recherches, responsable du Groupe Datation C2RMF, département Recherche (pascale.richardin@ culture. gouv.fr). Magali Coudert, collaboratrice scientifique de conservation-Égypte romaine et byzantine, Service du Récolement, direction de la Recherche et des Collections, musée du Louvre (magali.coudert@louvre.fr). 


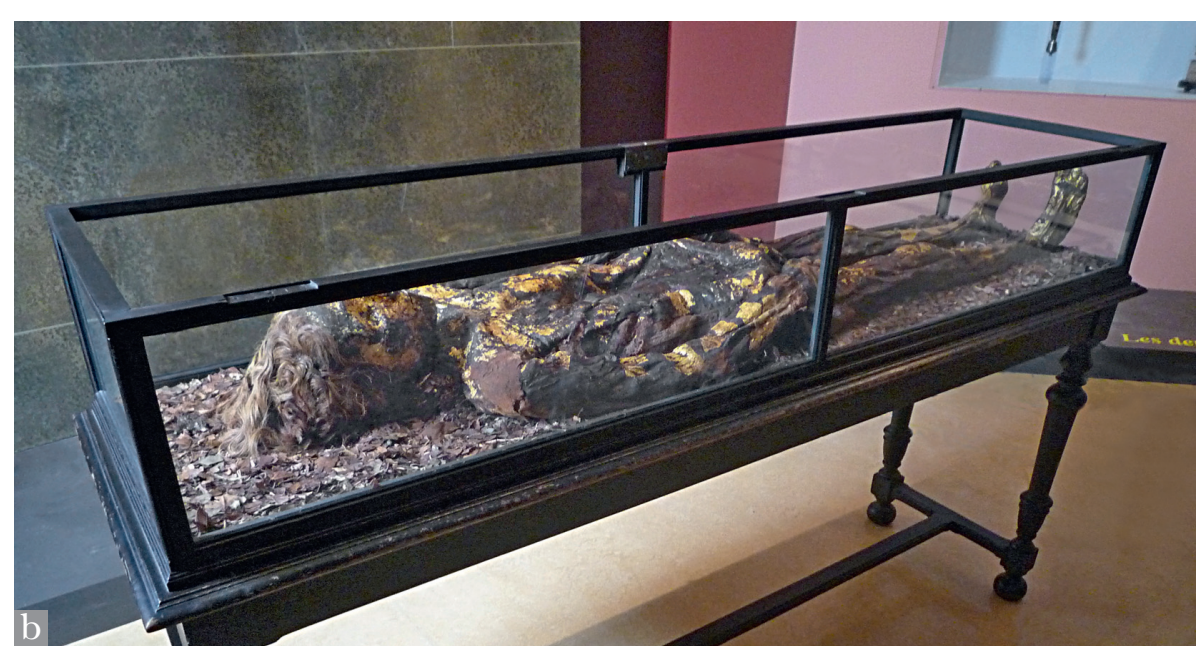

Fig. 1 b. La momie dorée de Dunkerque dans sa vitrine.

\section{Matériel et méthodes}

Nous avons réalisé plusieurs prélèvements sur la momie : un échantillon de cheveux prélevés sur la tête de la momie (ChD1 - $66 \mathrm{mg}$ ) et un autre déposé sur le lit de feuilles (ChD2 $45 \mathrm{mg}$ ). Afin de pouvoir vérifier si le feuillage était bien associé au corps de la momie, une feuille de mûrier a été collectée à côté du corps (FeuD - $160 \mathrm{mg}$ ), ainsi qu'un échantillon détaché de textile (TexD - $17 \mathrm{mg}$ ).

La préparation des échantillons a nécessité une étape préliminaire d'extraction, surtout pour le textile qui était très enduit de matières organiques (fig. $2 \mathrm{a}$ et $\mathrm{b}$ ). Le protocole consiste en une série de lavages avec un mélange dichlorométhane/méthanol (1:1 v/v), puis avec de l'acétone, suivie de nombreux rinçages à l'eau ultrapure. Cette méthode est proche de celle de BlighDyer ${ }^{10}$ mise en place pour l'extraction des lipides et utilisée en routine pour l'analyse de la matière organique archéologique à des fins d'analyses chroma-tographiques ${ }^{11}$.

Les échantillons végétaux (textiles et feuilles) subissent des traitements avec des solutions aqueuses d'acide chlorhydrique et de soude à $80^{\circ} \mathrm{C}$, alternés avec des rinçages à l'eau ultrapure jusqu'à neutralité des eaux de lavage (méthode acide/base/acide). La préparation des cheveux est fondée sur l'extraction sélective de la kératine du cortex ${ }^{12}$ par réduction à l'aide du dithiothréitol (ou DTT). Cela permet de s'affranchir des protéines de la cuticule, qui pourraient contenir des contaminations extérieures.

Tous les extraits séchés (textiles, végétaux ou kératine) subissent ensuite une combustion sous vide poussé et à haute température. Enfin, le dioxyde de carbone récupéré est réduit par de l'hydrogène en présence de poudre de fer à $600{ }^{\circ} \mathrm{C}$, au cours de la graphitisation. Le carbone graphite obtenu se
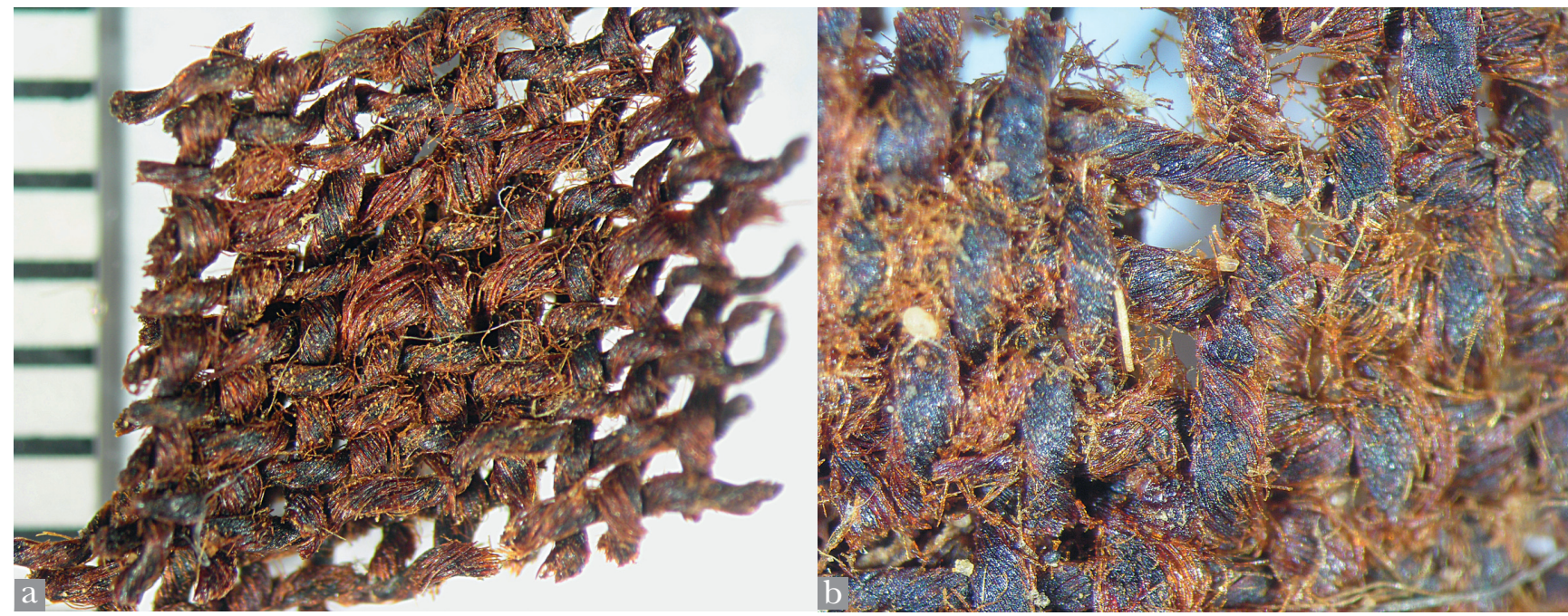

Fig. 2 a et $b$. Observation sous loupe binoculaire de l'échantillon de textile (TexD) montrant la quantité importante de matière organique. $\odot$ C2RMF/P. Richardin. 
Tableau 1. Résultats des âges radiocarbone et calibrés

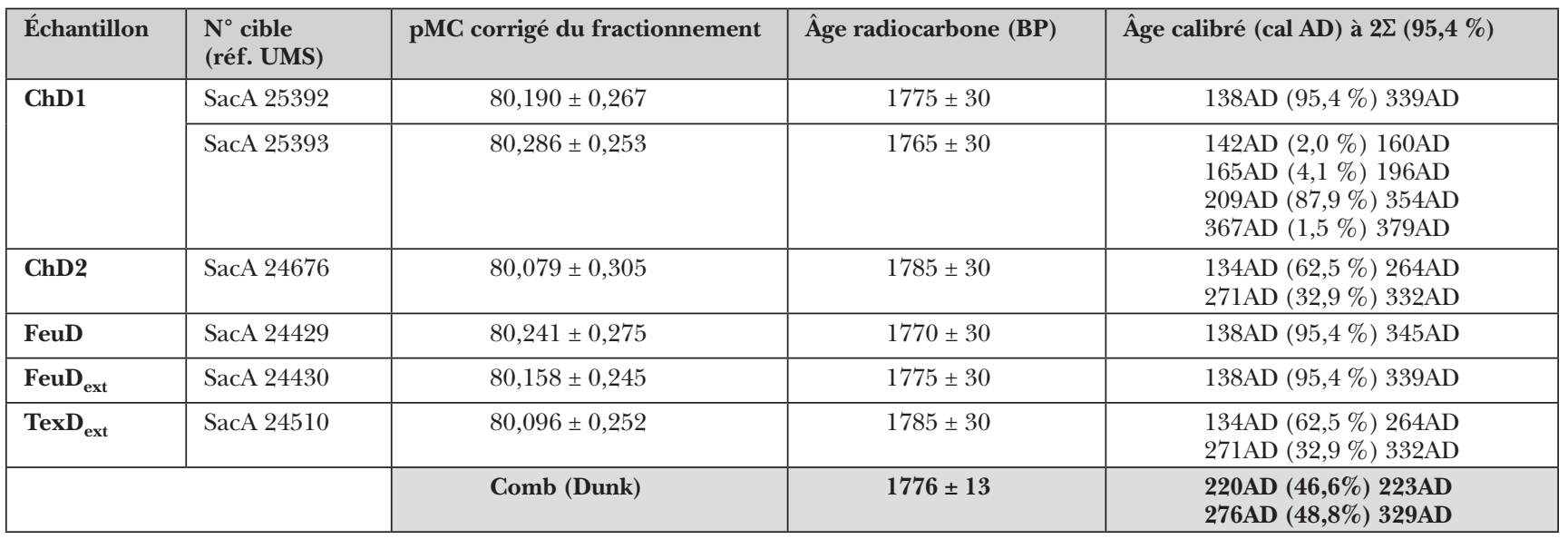

dépose sur la poudre de fer et l'ensemble est ensuite pressé dans une cible. Les mesures du carbone 14 ont été réalisées par AMS (spectrométrie de masse par accélérateur) sur l'accélérateur ARTEMIS, situé au CEA de Saclay ${ }^{13}$.

\section{Résultats}

Les âges radiocarbone ainsi que les âges calibrés ${ }^{14}$ pour chaque échantillon sont donnés dans le tableau 1.

La première remarque concerne la bonne reproductibilité du protocole d'extraction de la kératine. En effet, sur deux mesures indépendantes sur l'échantillon de cheveux ChD1 (préparation de l'échantillon et mesure), les dates obtenues sont identiques : $1775 \pm 30$ ans BP pour le premier essai et $1765 \pm 30$ ans BP pour le second.

L'innocuité des extractions avec les solvants pour la datation par le carbone 14 a déjà été montrée sur de nombreux exemples de bois ${ }^{15}$, de textiles ou de cheveux ${ }^{16}$. Nous l'observons ici avec les deux essais réalisés sur les feuilles de mûrier, qui n'étaient pas enduites de matières organiques. En effet, la date obtenue pour les feuilles extraites $\left(\mathbf{F e u D}_{\mathbf{e x t}}\right)$ et celle pour les feuilles non-extraites (FeuD) sont identiques : 1770 \pm 30 ans BP et $1775 \pm 30$ ans BP respectivement.

Nous avons vu que les deux échantillons de cheveux (ChD1 et ChD2) ont donné des résultats similaires (1775 \pm 30 ans BP, $1765 \pm 30$ ans BP pour ChD1 et $1785 \pm 30$ ans BP pour ChD2). Ainsi, l'échantillon prélevé sur le lit de feuilles appartient bien à la momie.

Tous les âges radiocarbone (cheveux ChD1 et ChD2, textile TexD et végétal FeuD) sont identiques et sont représentatifs du même événement. Ils peuvent donc être combinés, afin d'obtenir une date plus précise pour la momie et avec une marge d'erreur plus faible. La fonction R_Combine du logiciel OxCal 4.2.4 ${ }^{17}$ donne alors un âge radiocarbone de $1776 \pm 13$ ans BP, donc une date comprise entre 220 et 330 après J.-C.

\section{Conclusion}

Toutes les dates calibrées montrent que la momie dorée de Dunkerque se situe dans la période romaine de l'Égypte. Cette datation est confortée par l'application de feuilles d'or sur le corps de la momie, technique de traitement des corps régulièrement observée pour cette période.

Cet exemple confirme l'importance des protocoles de préparation des échantillons pour la datation par le carbone 14. L'extraction de la kératine du cortex des cheveux des momies permet d'obtenir des résultats fiables et reproductibles sur des cheveux avec des quantités de prélèvement qui peuvent se limiter à $20 \mathrm{mg}$. Cette méthode nous a permis par ailleurs de vérifier que des cheveux, prélevés auprès d'un corps momifié, n'appartenaient pas à la momie étudiée ${ }^{13}$, car la date obtenue était très éloignée de celle obtenue pour des cheveux prélevés sur la tête de la momie. La datation par le carbone 14, corrélée aux mesures isotopiques en carbone, azote et soufre obtenues sur des cheveux, est également essentielle pour mieux comprendre et interpréter l'évolution dans le temps de l'alimentation des populations ${ }^{18,19}$.

Bien sûr, ces résultats isolés ne sont utiles que s'ils sont confortés et/ou complétés par ceux obtenus dans d'autres disciplines (histoire et archéologie, anthropologie, paléopathologie, odontologie, etc.), d'où l'importance de la mise en place de projets pluridisciplinaires dans le cadre de l'étude des corps momifiés. C'est ce que ce premier projet nous a permis de réaliser, grâce à de nombreux programmes de recherche nationaux et internationaux sur l'analyse et/ou la datation de cheveux, poils ou fourrures présents sur des objets de musée. 


\section{Notes}

1. Hedges, 1992.

2. Beavan, 2012

3. Cadot, 2007.

4. Richardin, 2011.

5. Diaz-Zorita, 2016. Cadot, 2013.

Touzeau, 2014.

6. Quiles, 2014.

7. Cordonnier, 2013

8. Le SRDAI a effectué le récolement d'un ensemble d'environ 4500 œuvres provenant du site égyptien d'Antinoé. Ces pièces - parmi lesquelles subsistent aujourd'hui une quarantaine de momies ont été réparties par l'État au début du $\mathrm{Xx}^{\mathrm{e}}$ siècle à travers 70 institutions françaises.

À l'occasion de la publication de cette collection, Magali Coudert et Yannick Lintz ont mis en place un programme de recherche pluridisciplinaire sur l'ensemble des momies retrouvées, les corps égyptiens de cette période ayant rarement été étudiés

9. Richardin, 2013a. Richardin, 2013b. Benazeth, 2013.

10. Bligh, 1959.

11. Garnier, 2014.

12. Richardin, 2011.

13. Moreau, 2013.

14. Dès le début de la méthode de datation par le carbone 14, les chercheurs ont remarqué un désaccord avec les dates obtenues sur des objets archéologiques bien datés. Une des hypothèses théoriques de la méthode, qui était de dire que la teneur en ${ }^{14} \mathrm{C}$ dans l'atmosphère ne variait pas dans le temps, est bien entendu fausse. Pour pallier à cela, une courbe de calibration, réalisée grâce à d'autres techniques de datation qui ne dépendent pas du taux de ${ }^{14} \mathrm{C}$ dans l'atmosphère, comme la dendrochronologie et la datation par Uranium/Thorium de coraux par exemple, permet de transformation des âges radiocarbone en âges calendaires. Ces derniers - compris entre 0 à 50000 ans - peuvent être déterminés grâce à plusieurs logiciels en ligne sur internet et aux données de calibration les plus récentes.

15. Richardin, 2015

16. Richardin, 2016.

17. Bronk Ramsey, 1994.

18. Drucker, 2013.

19. Diaz-Zorita, 2016.

\section{Bibliographie}

Beavan N., Halcrow S., Mac Fadgen B. et al., 2012, "Radiocarbon dates from jar and coffin burials of the Cardamon Moutains reveal a unique mortuary ritual in Cambodia's Late to PostAngkor period (15th-17th centuries AD)", Radiocarbon 54(1), p. 1-22. Benazeth D., Richardin P., Gandolfo N., 2013, "L'apport de la recherche scientifique à la connaissance des tissus ", in Antinoé (Momies, tissus, céramiques et autres antiques) - Envois de l'État et dépôts du musée du Louvre (18951925) - dir. Yannick Lintz et Magali Coudert, éditions Musée du Louvre, p. 114-119.

Bligh E. G., Dyer W. J., 1959, “A rapid method for total lipid extraction and purification", Canadian Journal of Physiology and Pharmacology 37, p. 911-917.

Bronk Ramsey C., 1994, "Analysis of Chronological Information and Radiocarbon Calibration : The Program OxCal", Archaeological Computing Newsletter 41, p. 11-16.

Cadot L., 2007, « Les restes humains : une gageure pour les musées? ", La Lettre de l'OCIM 109, 5-15.

Cadot L., Coudert M., Dal Prà P., Gandolfo N., Langlois J., Richardin P., Timbart N., Weygand S., 2013, " Étude et restauration de deux momies du musée Joseph-Denais de Beaufort-en-Vallée ", Technè, $\mathrm{n}^{\circ} 38$, p. 58-64.

Cordonnier A., 2013, Dunkerque et la collection d'Antinoé, cité de l'Égypte Antique, Dunkerque, éditions Musée des BeauxArts.

Diaz-Zorita M., Drucker D., Richardin P., Silva- Pinto V., Sepúlveda M. Bocherens H., 2016, "Marine food consumption in coast northern Chilean (Atacama Desert) population during the Formative Period: Implication of isotopic evidence $(\mathrm{C}, \mathrm{N}, \mathrm{S})$ for Neolithic process in south central Andes", Journal of Archaeological Sciences, 6, p. 768-776.

Drucker D., Bocherens H., 2013, « Reconstitution de l'alimentation de la population d'Antinoé aux époques romaine, byzantine et islamique par les analyses d'isotopes stables du carbone, de l'azote et du soufre de la kératine de cheveux de momies ", in Antinoé (Momies, tissus, céramiques et autres antiques) - Envois de l'État et dépôts du musée du Louvre (1895-1925) dir. Yannick Lintz et Magali Coudert, éditions Musée du Louvre, p. 82-88.

Garnier N., 2014, " Méthodologies d'analyse chimique organique en archéologie”, in ArchaeoAnalytics - Chromatography and DNA analysis in archaeology, p. 13-39.

Hedges R. E. M., Vanklinken G.J., 1992, "A review of current approaches in the pretreatment of bone for radiocarbon dating by AMS", Radiocarbon 34(3), p. 279-291

Moreau C., Caffy I., Comby C., Delqué-Kolic E., Dumoulin J.-P., Hain S., Quiles A., Setti V., Souprayen C., Thellier B., Vincent J., 2013, "Research and development of the Artemis 14C AMS Facility: status report", Radiocarbon $55(2-3)$, p. 331-377.

Quiles A., Delqué-Količ E., Bellot-Gurlet L., Comby-Zerbino C., Ménager M., Paris C., Souprayen C., Vieillescazes C., Andreu-Lanoë G., Madrigal K., 2014, "Embalming as a Source of Contamination for Radiocarbon dating of Egyptian Mummies: On a New Chemical Protocol to Extract Bitumen", Archéosciences, 38(1), p. 135-149.

Richardin P., Gandolfo N., Carminati P., Walter P., 2011, "New protocol for radiocarbon dating of hair and keratin type samples - Application to an Andean mummy from the National Museum of Natural History in Paris", Archaeological and Anthropological Sciences 3(4), p. 379-384.

Richardin P., Coudert M., Gandolfo N., Vincent J., 2013a, "Radiocarbon dating of mummified human remains Application to a series of Coptic mummies from the Louvre Museum”, Radiocarbon 55(3-4), p. 345-352.

Richardin P., Coudert M., Gandolfo N., Vincent J., 2013b, « Datation par le radiocarbone : Étude chronologique des momies et de leur matériel funéraire ", in Antinoé (Momies, tissus, céramiques et autres antiques) - Envois de l'État et dépôts du musée du Louvre (1895 1925) - dir. Yannick Lintz et Magali Coudert, éditions Musée du Louvre, p. 74-81.

Richardin P., Lavier C., Horta H., Figueroa V., Lira N., 2015, "Radiocarbon dating of Atacama (Chile) snuffs trays: an update on stylistic and chronological correlations", Radiocarbon 57(5), p. 1-10.

Richardin P., Perraud A., Hertzog Y., Madrigal K., Berthet D., 2016,

"Radiocarbon dating of a series of Egyptian mummies heads from Confluences Museum, Lyon (France)”, in Radiocarbon (sous presse).

Touzeau A., Amiot R., Blichert-Toft J., Flandrois J.-P., Fourel F., Gabolde L., Grossi V., Martineau F., Richardin P., Lécuyer C., 2014, "Diet of ancient Egyptians inferred from stable isotope systematics", Journal of Archaeological Science 46, p. 114-124. 